\title{
PROTOTYPING OF DSSDS FOR PARTICLE TRACKING AND SPECTROSCOPY WITHIN THE EXL PROJECT AT FAIR
}

\author{
L. X. CHUNG \\ Institute for Nuclear Science and Technology \\ and \\ GSI, Helmholtzzentrum für Schwerionenforschung GmbH, Darmstadt, Germany \\ N. T. KHAI \\ Institute for Nuclear Science and Technology \\ P. EGELHOF, S. ILIEVA, AND B. STREICHER \\ GSI, Helmholtzzentrum für Schwerionenforschung GmbH, Darmstadt, Germany \\ R. BORGER, C. RIGOLLET, N. KALANTAR-NAYESTANAKI, AND A. NAJAFI \\ KVI, University of Groningen, the Netherlands \\ T. DAVINSON AND P. J. WOODS \\ University of Edinburgh, United Kingdom \\ V. EREMIN \\ PTI, Ioffe Physical Technical Institute of Russian Academy of Sciences, \\ St. Petersburg, Russia \\ M. MUTTERER \\ GSI, Helmholtzzentrum für Schwerionenforschung GmbH, Darmstadt, Germany \\ and \\ Institut für Kernphysik, Technische Universität Darmstadt, Darmstadt, Germany \\ M. VON SCHMID \\ Institut für Kernphysik, Technische Universität Darmstadt, Darmstadt, Germany
}

\begin{abstract}
Prototype double-sided silicon strip detectors (DSSDs) of $300 \mu \mathrm{m}$ thickness produced at PTI St. Petersburg (Russia) were tested for the use as position sensitive, $\Delta E$ and E detectors for tracking and particle identification in the EXL (EXotic nuclei studied in Light-ion induced reactions at the NESR storage ring) setup at the FAIR (Facility for Antiproton and Ion Research) project at GSI. We describe the characteristics of detectors with $16 \times 16,64 \times 64$ and $64 \times 16$ strips, respectively. The response of these detectors for ${ }^{241} \mathrm{Am} \alpha$ particles injected either from the $p$ or $n$ side was examined. The test measurements were performed partially at GSI and the University of Edinburgh. A first in-beam test with a proton beam of $50 \mathrm{MeV}$ with the latter two DSSDs and two $6.5 \mathrm{~mm}$ thick Si(Li) detectors was also done at KVI Groningen, the Netherlands. The results reveal good spectroscopic properties of these detectors.
\end{abstract}




\section{INTRODUCTION}

The objective of the EXL-project, which is part of the NUSTAR (Nuclear STructure, Astrophysics and Reactions) program at FAIR [1], is to capitalize on light-ion induced direct reactions in inverse kinematics using novel storage ring techniques. For the key physics issues addressed in [2], a complex detection system must satisfy the demand of a highly efficient, high-resolution universal system and to be applicable to a wide class of reactions.

Double-sided silicon detectors are a major part of the EXL silicon particle array (ESPA) [2] which, surrounding a gas-jet or hydrogen droplet target, will be used as a position sensitive integrated $\Delta \mathrm{E}-\mathrm{E}$ detector array. It is dedicated to light recoil particle ( $\mathrm{p}$, alpha) detection in the wide energy range from about $100 \mathrm{keV}$ to nearly $200 \mathrm{MeV}$. These detectors should have low detection threshold, high energy resolution and detection efficiency and, possibly, pulse shape discrimination (PSD) capability for particle identification at low energies.

This paper presents first results on the development of spectroscopic DSSDs for the EXL experiment which have proper design: moderate pitch size, large strip width, small interstrip gap and a thin entrance p-side window in order to reach the above requirements. The detectors were produced in PTI St. Petersburg (Russia) under INTAS-GSI project \#06-1000012-8844.

\section{DETECTORS}

The prototype DSSDs studied have a thickness of $300 \mu \mathrm{m}$ and $16 \times 16,64 \times 64$ and $64 \times 16$ strips. The smallest sensor has 16 strips at each side and is $7.1 \times 7.1 \mathrm{~mm}^{2}$ in area. On the p-side, the widths of strips and interstrip gaps are $285 \mu \mathrm{m}$ and $15 \mu \mathrm{m}$, respectively, while these values are $235 \mu \mathrm{m}$ and $65 \mu \mathrm{m}$ on the n-side. Thus, on both sides a $300 \mu \mathrm{m}$ wide pitch is formed. There are 64 strips at each side of the bigger $64 \times 64$ DSSD at an area of $21.2 \times 21.2 \mathrm{~mm}^{2}$. The sensor strips have the same configuration as in the small detector. The second big detector tested is the $64 \times 16$ DSSD which has the same outer dimension as the $64 \times 64$ DSSD. The only difference between them is that at the n-side there are 16 strips with a pitch size of $1200 \mu \mathrm{m}$ formed by $1135 \mu \mathrm{m}$ wide strips and $65 \mu \mathrm{m}$ interstrip gaps. The characteristic features of the three DSSDs are summarized in table 1.

Fig. 1 is a schematic view of the structures of both DSSD faces processed on to a $300 \mu \mathrm{m}$ thick n-bulk wafer. On the front side (p-side) of all DSSDs, the strips are separated by a gap passivated by $\mathrm{SiO}_{2}$ layer and, besides an approximately $400 \mathrm{~nm}$ thick $\mathrm{p}^{+}$implantation layer windowless. The electric contact is realized through a $800 \mathrm{~nm}$ thick and $17.5 \mu \mathrm{m}$ wide aluminum frame. The strips at the rear side (n-side) are entirely covered with aluminum. The isolation between them is established by a $450 \mathrm{~nm}$ thick $\mathrm{p}^{+}$ implantation. There are 5 guard rings surrounding the DSSDs. The bias voltage is applied to the innermost ring serving as bias ring (punch-through biasing).

\section{MEASUREMENT AND DATA ANALYSIS}

In the EXL experiment particles will always impact perpendicularly on the surface of the DSSDs and be injected from the p-side. As mentioned above, the DSSDs will be 
Table 1. DSSD characteristic features (in $\mu \mathrm{m}$ )

\begin{tabular}{|l|l|l|l|l|l|l|}
\hline \multirow{2}{*}{ DSSD } & \multicolumn{3}{|l|}{ p-side } & \multicolumn{3}{l|}{ n-side } \\
\cline { 2 - 7 } & Strip & Gap & Pitch & Strip & Gap & Pitch \\
\hline $16 \times 16$ & 285 & 15 & 300 & 235 & 65 & 300 \\
\hline $64 \times 64$ & 285 & 15 & 300 & 235 & 65 & 300 \\
\hline $64 \times 16$ & 285 & 15 & 300 & 1135 & 65 & 1200 \\
\hline
\end{tabular}

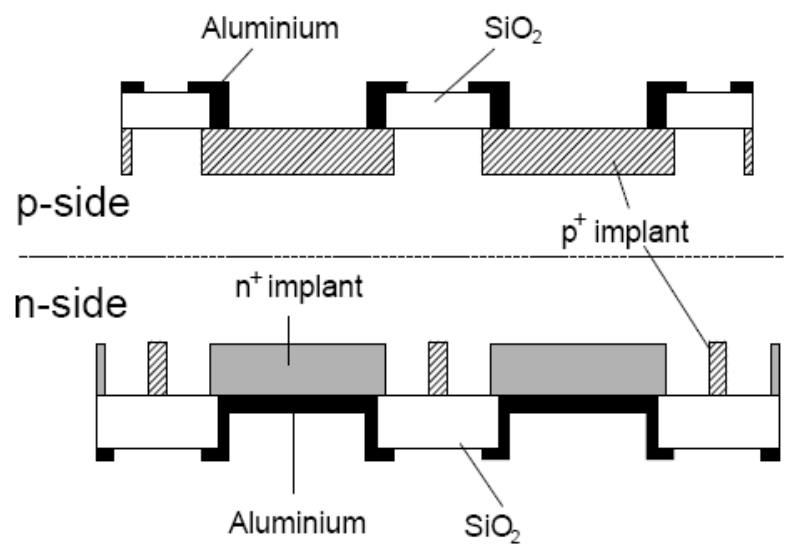

Fig. 1. P-side and n-side cross section of $16 \times 16$ DSSD (n-side strips are rotated by $90^{\circ}$ for illustration)

dedicated to light particle detection with energy range spreading from about $100 \mathrm{keV}$ to nearly $200 \mathrm{MeV}$. Therefore, in the test experiment, an alpha source and a $50 \mathrm{MeV}$ proton beam were used.

The ${ }^{241} \mathrm{Am}-\alpha$ particles with $5.5 \mathrm{MeV}$ energy are stopped in the detector at a penetration depth of $27 \mu \mathrm{m}$. This low-energy particles allowed to explore the DSSD wafer's structure and the influence in charge collection towards strips both, with p-side and n-side injection. Because of the current limitation in read-out electronics to 32 channels only the $16 \times 16$ DSSDs and the $64 \times 16$ DSSDs with 4 adjacent strips coupled together at the 64 -strip side were tested at GSI. The $64 \times 64$ DSSD test was performed at the University of Edinburgh where electronics equipped with 128 read-out channels was available.

Fig. 2 displays the electronic scheme of the test experiments at GSI. The total depletion voltage was established at $-50 \mathrm{~V}$, but an overbias of up to $-200 \mathrm{~V}$ was applied to the p-side with AC- coupled preamps. The ground potential was connected to the n-side through DC-coupled preamps. The signals of the strips on the front side were connected to channel 1 to 16, and the rest (17-32 channels) was used for the strip signals from the rear side. The DAQ recorded events only when the energy signal was coincident with the gate produced as OR of all timing signals from front side. This trigger was kept in both $\mathrm{p}$ - and n-side injection measurement discussed in the next section.

A second in-beam test stage was done with a $50 \mathrm{MeV}$ proton beam at KVI Groningen. The $64 \times 64$ and $64 \times 16$ DSSDs were chosen. The experimental setup is shown in 


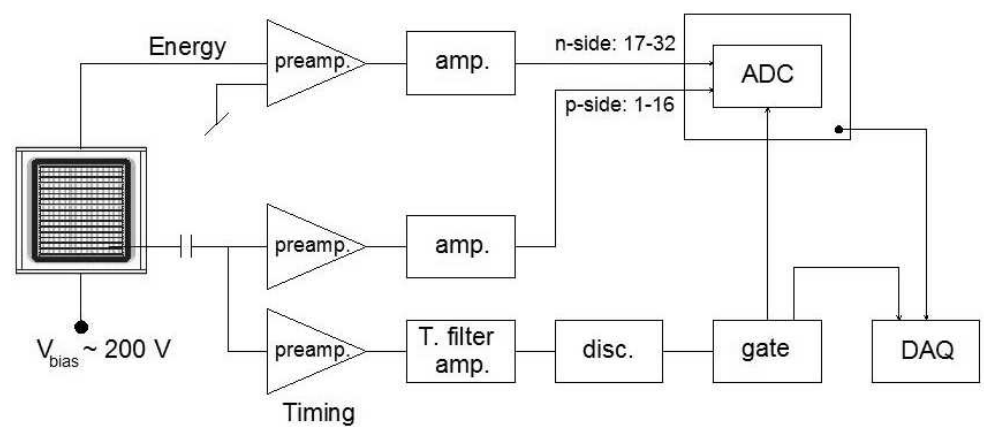

Fig. 2. The electronic scheme of GSI test experiments

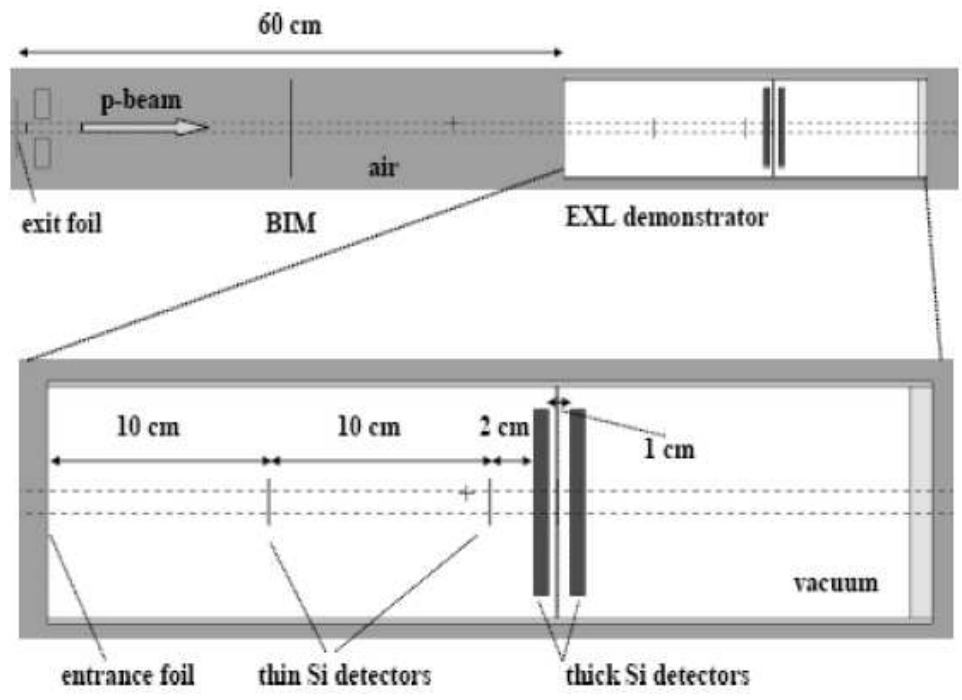

Fig. 3. KVI experimental set up, two DSSDs and two $\mathrm{Si}(\mathrm{Li})$ s were placed in vacuum chamber.

Fig. 3. The protons punched through both DSSDs and were stopped in the second $\mathrm{Si}(\mathrm{Li})$ detector [3].

\section{RESULTS AND DISCUSSION}

\section{IV.1. Tests with alpha source}

Fig. 4 shows the peaks of the alpha source. With p-side injection, the FWHMs for $5.5 \mathrm{MeV} \alpha$ particles were between $14 \mathrm{keV}$ and $26 \mathrm{keV}$ for both, p- and n-side energy spectra of the small detector (panel a and b), dependent on electronic settings. The five energies of ${ }^{241} \mathrm{Am}$ are separated. In contrast, these energies are no longer resolved in the case of $\mathrm{n}$-side injection (panel c and d). The energy resolution is about $50 \mathrm{keV}$ due to the aluminum metallic layer at the entrance of the n-side strips (Fig. 1). 

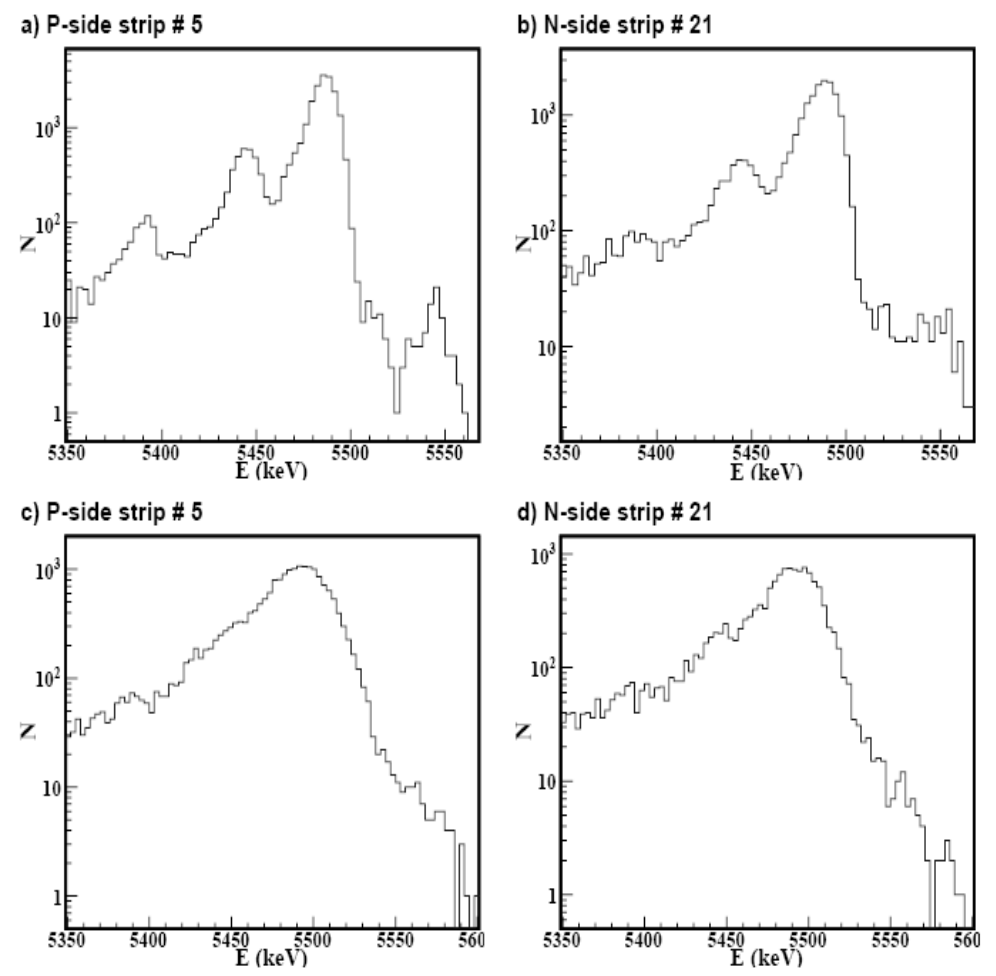

Fig. 4. Energy spectrum of $16 \times 16$ DSSD. a) and b): p-side injection, c) and d): n-side injection

A characteristic energy spectrum is displayed in Fig. 5 which is taken from strip \#5 at the p-side. The spectrum consists of 3 regions: main peaks (of ${ }^{241} \mathrm{Am}$ source), shifted peaks (energy lost by metallic frame, by the partial overlap of bonding pads or by inducing positive signal in adjacent strips) and the continuous background region due to interstrip events. Each strip energy spectrum consists of the contributions from all event types discussed below.

The performance of the $64 \times 64$ DSSD measured at the University of Edinburgh with different electronics was very similar to that of the $16 \times 16$ DSSD. Measured energy resolutions were 29 and $32 \mathrm{keV}$ for energy spectra of the strips on p- and n-side, respectively.

High efficiency is an important requirement for DSSDs in the EXL setup. To determine this information, we plotted a correlation between the energy signals recorded on pand n-side strips, respectively. Then, the scatter plot (Fig. 6) was divided into 4 regions corresponding to 4 event types: (1) strip-strip, (2) strip-interstrip, (3) interstrip-strip and (4) interstrip-interstrip. Where, 'strip' means the signal was created by the incident particle only on one strip, while 'interstrip' means the signal was shared between 2 neighboring strips. The DSSDs' efficiency is tabulated in table 2. This gives the relative efficiency of each event type in the total detected event. 


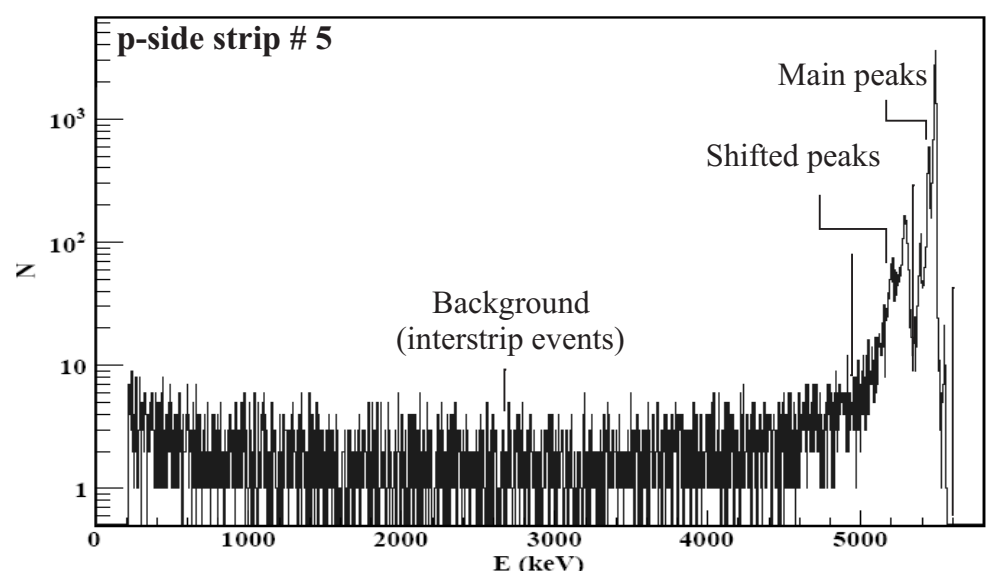

Fig. 5. Characteristic energy spectrum of $16 \times 16$ DSSD, p-side injection

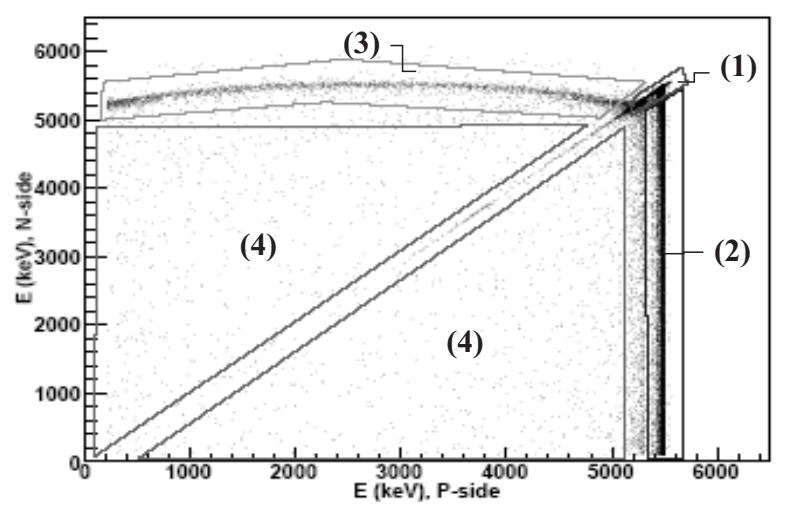

Fig. 6. 2-D energy signal correlation between p- and n-side, p-side in jection

One possible explanation for the bending in region (3) in Fig. 6 is a certain recombination and trapping probability of electrons in the interstrip region due to positive charges created at the $\mathrm{Si}_{-} \mathrm{SiO}_{2}$ interface. Consequently, the amount of collected charges toward the n-side is reduced. In addition, these positive charges would create locally a field inversion which would modify charge collection in the interstrip zone and make the situation dependent on particle penetration depth [4].

Table 2. DSSD Efficiency (\%), $5.5 \mathrm{MeV}$ alpha particles

\begin{tabular}{|l|l|l|}
\hline Event type & $16 \times 16$ & ${ }^{*} 64 \times 16$ \\
\hline$(1)$ & 76.8 & 93.9 \\
\hline$(2)$ & 18.2 & 6.1 \\
\hline$(3)$ & 4.1 & $\sim 0.0$ \\
\hline$(4)$ & 0.9 & $\sim 0.0$ \\
\hline
\end{tabular}



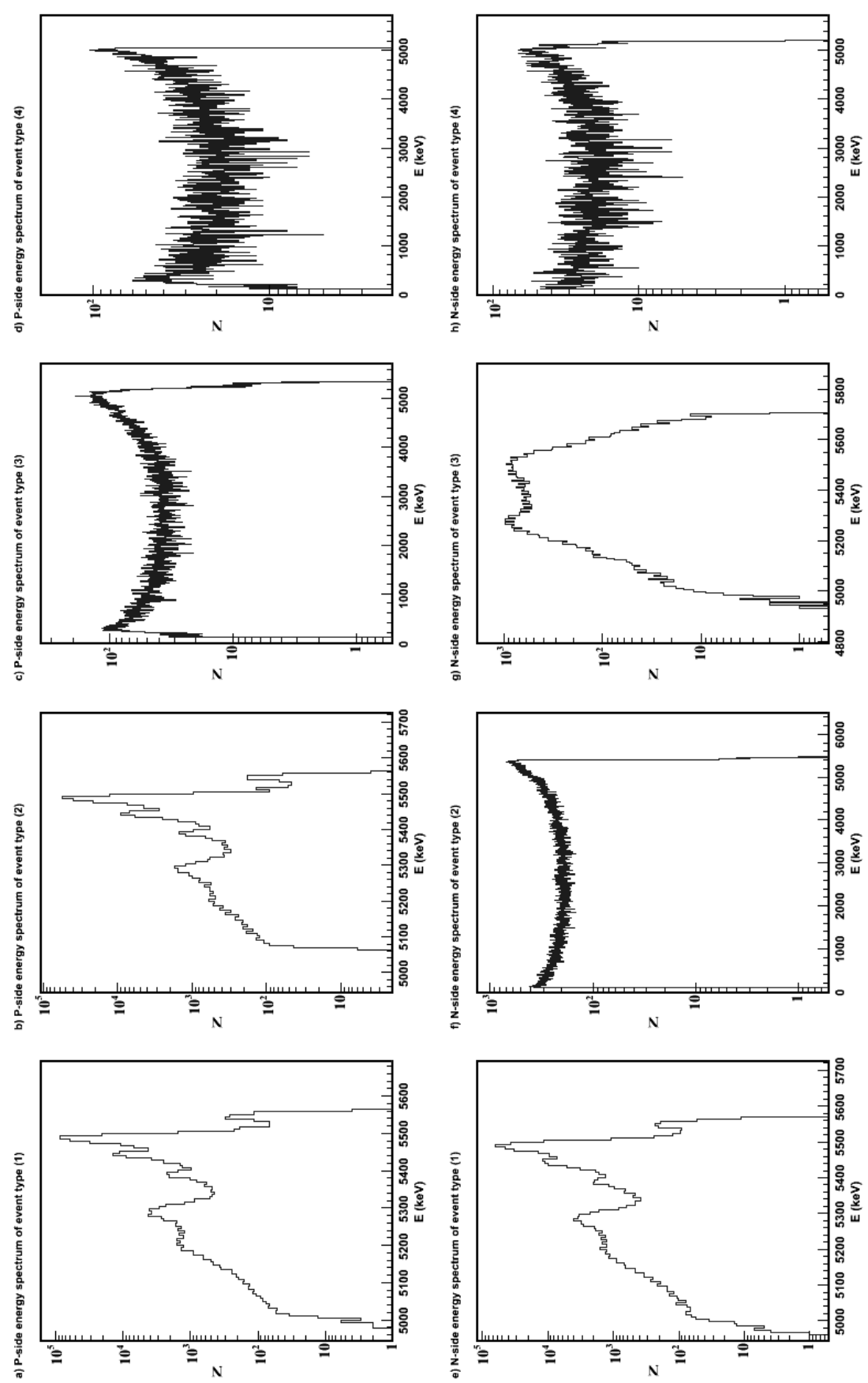

Fig. 7. P- and n- side energy spectra of 4 event types, detail is explained in text. 
The front and rear side energy spectra of all event types are shown in Fig. 7. Panels $\mathrm{a}, \mathrm{b}$, e and $\mathrm{g}$ are the energy spectra of events induced signal in only one strip in one DSSD side. They contribute to the strip spectrum (Fig. 5) as the main and shifted peak regions. Except panel g, panels a, b and e show good energy resolution (FWHM=14 $\div 26 \mathrm{keV}$ ). The main peaks of the $\alpha$-source are separated. The rest panels in Fig. 7 present the energy spectra of events which gave energy sharing between 2 adjacent strips. In the strip spectrum, these appear as the continuous background region. The correlation of sharing energy E1 and E2, where E1 and E2 are the energy signals of 2 neighboring strips at one side of the DSSD, is plotted in Fig. 8. Comparing with Fig. 11 in [4], because our ADCs were not able to measure signals with both polarities, we couldn't see (in panel a) the parts of the signals with a reduction of total energy and of inverse signals in adjacent strips. In addition, since our DSSD's interstrip gap is small $(15 \mu \mathrm{m})$ the energy correlation of the detector entrance face strips does possibly not consist of the parts related with particles impinging on the region of field inversion. Panel $b$ shows a very similar pattern of the energy correlation of exit face strips to Fig. 13 in [4]. The reconstructed energy spectra $(\mathrm{E} 1+\mathrm{E} 2)$ in $\mathrm{p}-$ and $\mathrm{n}$-side are displayed in Fig. 9.
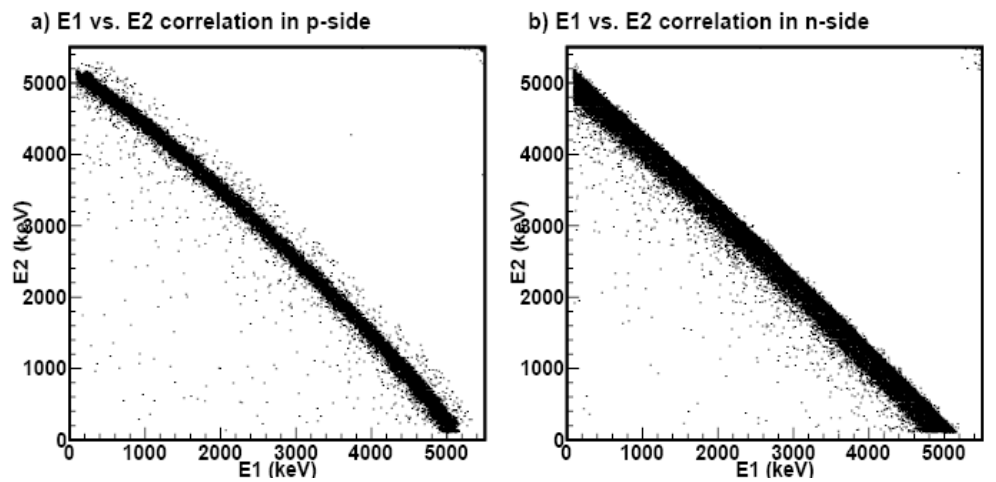

Fig. 8. E1, E2 correlation, p-side injection

The original energy of the incident particles when signals are induced at 2 adjacent strips is equivalent to the sum of E1 and E2. Except the outermost ones, each strip has two neighbors. The percentages of interstrip events were calculated by half the ratio of interstrip and strip count rates. The results were approximately $5 \%$ and $17 \%$ for strips at the p- and n-side, respectively (Fig. 10.a), consistent with the geometry of the $16 \times 16$ DSSD (Table 1). For n-side injection, the results were about $21 \%$ and $0.3 \%$ (Fig. 10.b). The response of the DSSDs with rear side injection is not fully understood and still needs more investigation.

\section{IV.2. Test with proton beam}

The second test phase of the DSSDs was done in an EXL demonstrator experiment at KVI Groningen with a $50 \mathrm{MeV}$ proton beam [5]. The experimental setup is shown in Fig. 3. It was the aim to study the DSSD response for punching through high-energy particles and to check the quality of total energy reconstruction of a telescope setup. 

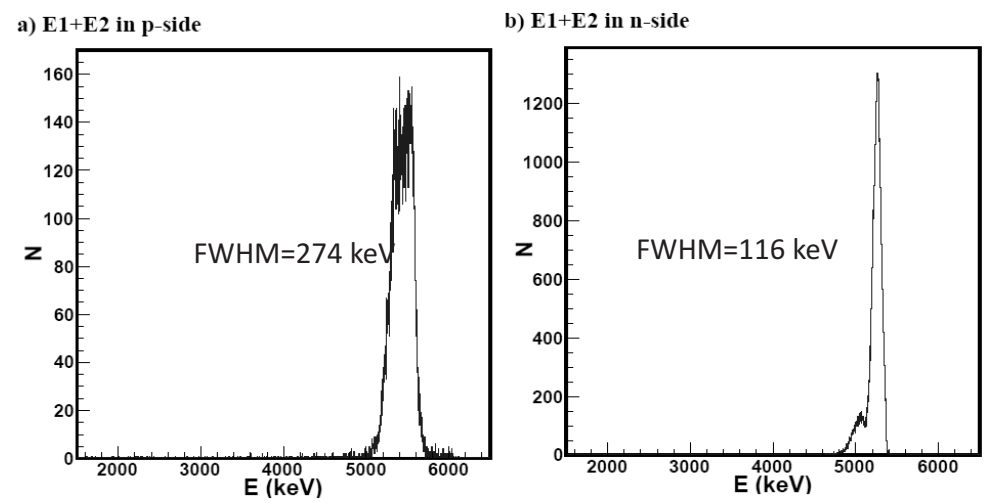

Fig. 9. E1+E2 spectra, p-side injection
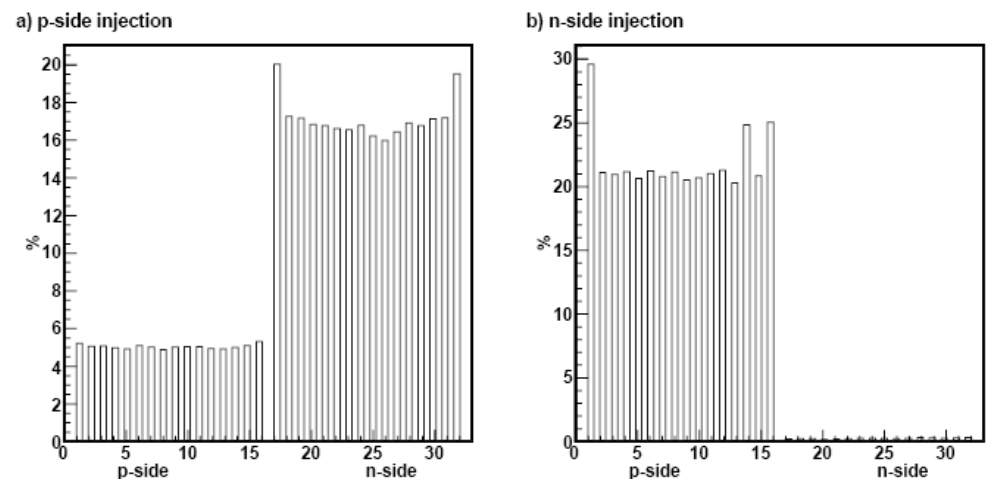

Fig. 10. Percentage of interstrip events of the $16 \times 16$ DSSD

The response of the DSSDs to punching through proton is shown in Fig. 11. The peaks are located at about $700 \mathrm{keV}$ with $\sim 200 \mathrm{keV}$ FWHM for strip energy spectra of $64 \times 64$ and $64 \times 16$ DSSDs.

The same 2-D energy signal correlation method as shown in Fig. 6 was used to determine the DSSD efficiency (Fig. 12). The results for the efficiencies are given in table 3. The reconstructed energy of the beam protons is summed up from the energy losses in the 2 DSSDs and the first $\mathrm{Si}(\mathrm{Li})$ and the residual energy in the second $\mathrm{Si}(\mathrm{Li})$. The FWHM for about 45.2 MeV total energy was determined to be $396 \mathrm{keV}$ (Fig. 13).

Table 3. DSSD Efficiency (\%), $50 \mathrm{MeV}$ proton beam

\begin{tabular}{|l|l|l|}
\hline Event type & $64 \times 64$ & $64 \times 16$ \\
\hline$(1)$ & 91.3 & 82.6 \\
\hline$(2)$ & 3.1 & 15.0 \\
\hline$(3)$ & 3.3 & 2.4 \\
\hline$(4)$ & 2.3 & $\sim 0.0$ \\
\hline
\end{tabular}




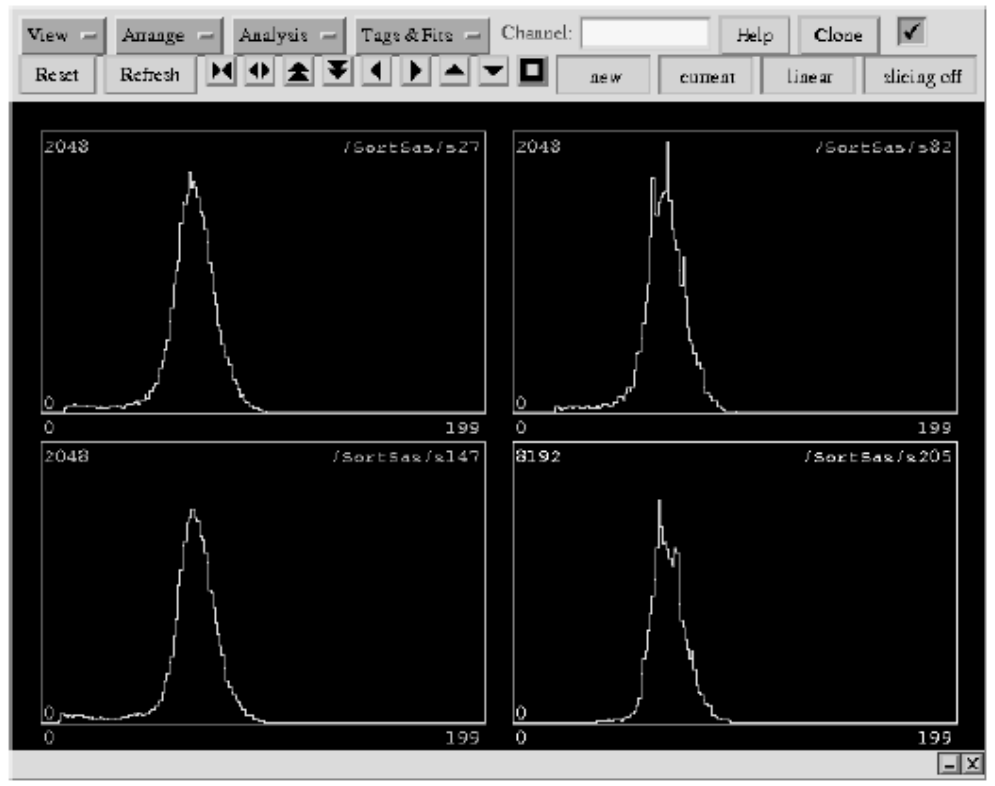

Fig. 11. Energy spectra of the DSSDs. $64 \times 64$ DSSD p-side spectrum - top left, n-side spectrum - top right; $64 \times 16$ DSSD p-side spectrum - bottom left; n-side pectrum - bottom right.

\section{(1): strip-strip}

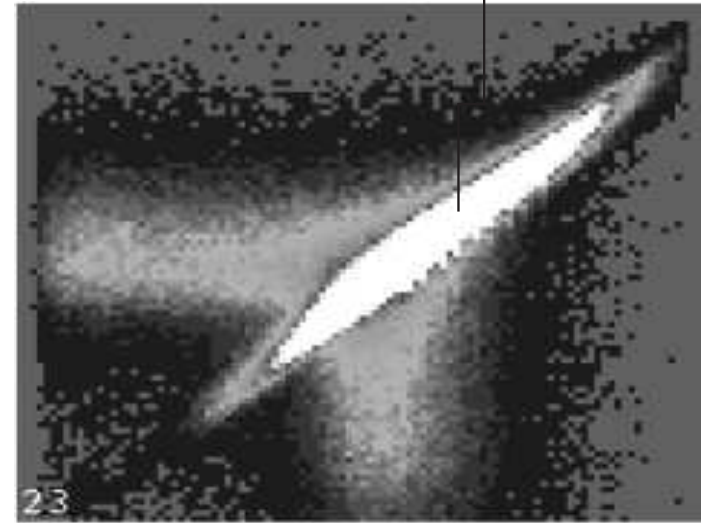

Fig. 12. 2-D energy signal correlation between p- and n-side of $64 \times 64$ DSSD

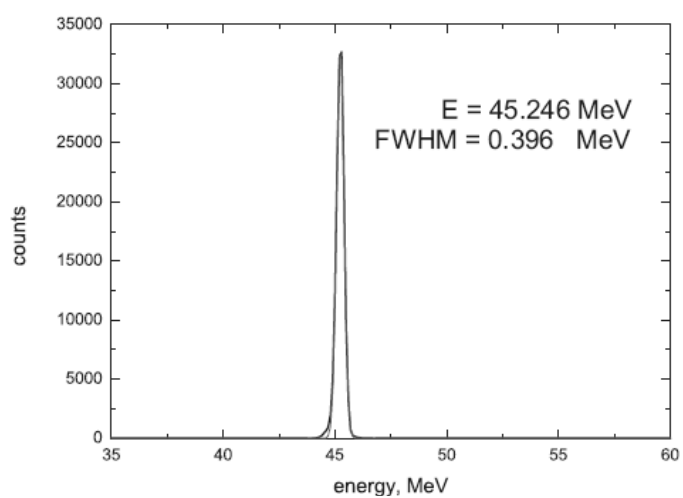

Fig. 13. Energy reconstruction for single proton events

\section{SUMMARY AND OUTLOOK}

Within an R\&D program using prototype detectors for the EXL project, several DSSDs were tested with $\alpha$ particle injected from both sides. With p-side injection, the result shows good spectroscopic properties of the detectors. The best resolution is $14 \mathrm{keV}$ FWHM for the p-side strip response of $5.5 \mathrm{MeV}$ alpha particle energy. The efficiency 
of the DSSDs is good with the main contribution from strip-strip events. The interstrip behavior conforms with the detector geometry. The DSSD performance with n-side injection becomes worse and this case still needs more investigation. For a demonstrator detector telescope consisting of DSSDs and $\mathrm{Si}(\mathrm{Li})$ s bombarded with $50 \mathrm{MeV}$ protons the total energy was reconstructed with $396 \mathrm{keV}$ FWHM resolution which is already close to the designed value. The possibility of pulse shape discrimination with the DSSDs has been investigated [6]. Also, the larger $64 \times 64$ DSSD chips will be tested for their capability of operation in the ultra-high vacuum environment of a storage ring.

\section{ACKNOWLEDGMENTS}

The authors L. X. Chung and N. T. Khai would like to thank Vietnam National Foundation for Science and Technology Development (NAFOSTED) for the support under grant number 103.01-2011.17.

\section{REFERENCES}

[1] Facility for Antiproton and Ion Research, http://www.fair-center.com.

[2] http://www-linux.gsi.de/ wwwnusta/tech_report/05-exl.pdf.

[3] http://www.fair-center.de/Publikationen.171.0.html? \&0=\&L=1.

[4] http://www.gsi.de/informationen/wti/library/scientificreport2006/PAPERS/FAIREXPERIMENTS-31.pdf

[5] Y. Blumenfeld et al., Nucl. Instr. and Meth. in Phys. Res. A421 (1999) 471.

[6] B. Streicher et al., GSI Scientific Report 2009, p 36.

[7] M. von Schmid et al., Nucl. Instr. and Meth. in Phys. Res. A629 (2011) 197-201

Received 14 March 2012. 